\title{
Erratum
}

\section{Erratum to “Automatic Segmentation of Ultrasound Tomography Image"}

\author{
Shibin Wu, ${ }^{1,2}$ Shaode Yu, ${ }^{1,2}$ Ling Zhuang, ${ }^{3}$ Xinhua Wei, ${ }^{4}$ Mark Sak, ${ }^{5,6}$ Neb Duric, ${ }^{5,6}$ \\ Jiani Hu, ${ }^{7}$ and Yaoqin $\mathrm{Xie}^{1}$ \\ ${ }^{1}$ Institute of Biomedical and Health Engineering, Shenzhen Institutes of Advanced Technology, Chinese Academy of Sciences, \\ 1068 Xueyuan Avenue, Shenzhen University Town, Shenzhen 518055, China \\ ${ }^{2}$ Shenzhen Colleges of Advanced Technology, University of Chinese Academy of Sciences, 1068 Xueyuan Avenue, \\ Shenzhen University Town, Shenzhen 518055, China \\ ${ }^{3}$ Department of Radiation Oncology, Northwestern Medicine Lake Forest Hospital, Lake Forest, IL 60045, USA \\ ${ }^{4}$ Department of Radiology, Guangzhou First Hospital, Guangzhou Medical University, Guangzhou 510180, China \\ ${ }^{5}$ Department of Oncology, The Karmanos Cancer Institute, Wayne State University, Detroit, MI 48201, USA \\ ${ }^{6}$ Delphinus Medical Technologies, Inc., Plymouth, MI 48170, USA \\ ${ }^{7}$ Department of Radiology, Wayne State University, Detroit, MI 48201, USA \\ Correspondence should be addressed to Yaoqin Xie; yq.xie@siat.ac.cn
}

Received 12 October 2017; Accepted 24 October 2017; Published 5 November 2017

Copyright (c) 2017 Shibin Wu et al. This is an open access article distributed under the Creative Commons Attribution License, which permits unrestricted use, distribution, and reproduction in any medium, provided the original work is properly cited.

In the article titled "Automatic Segmentation of Ultrasound Tomography Image" [1], the affiliation of the third author was incorrect. The corrected authors' list and affiliations are shown above.

\section{References}

[1] S. Wu, S. Yu, L. Zhuang et al., "Automatic segmentation of ultrasound tomography image," BioMed Research International, vol. 2017, Article ID 2059036, 8 pages, 2017. 

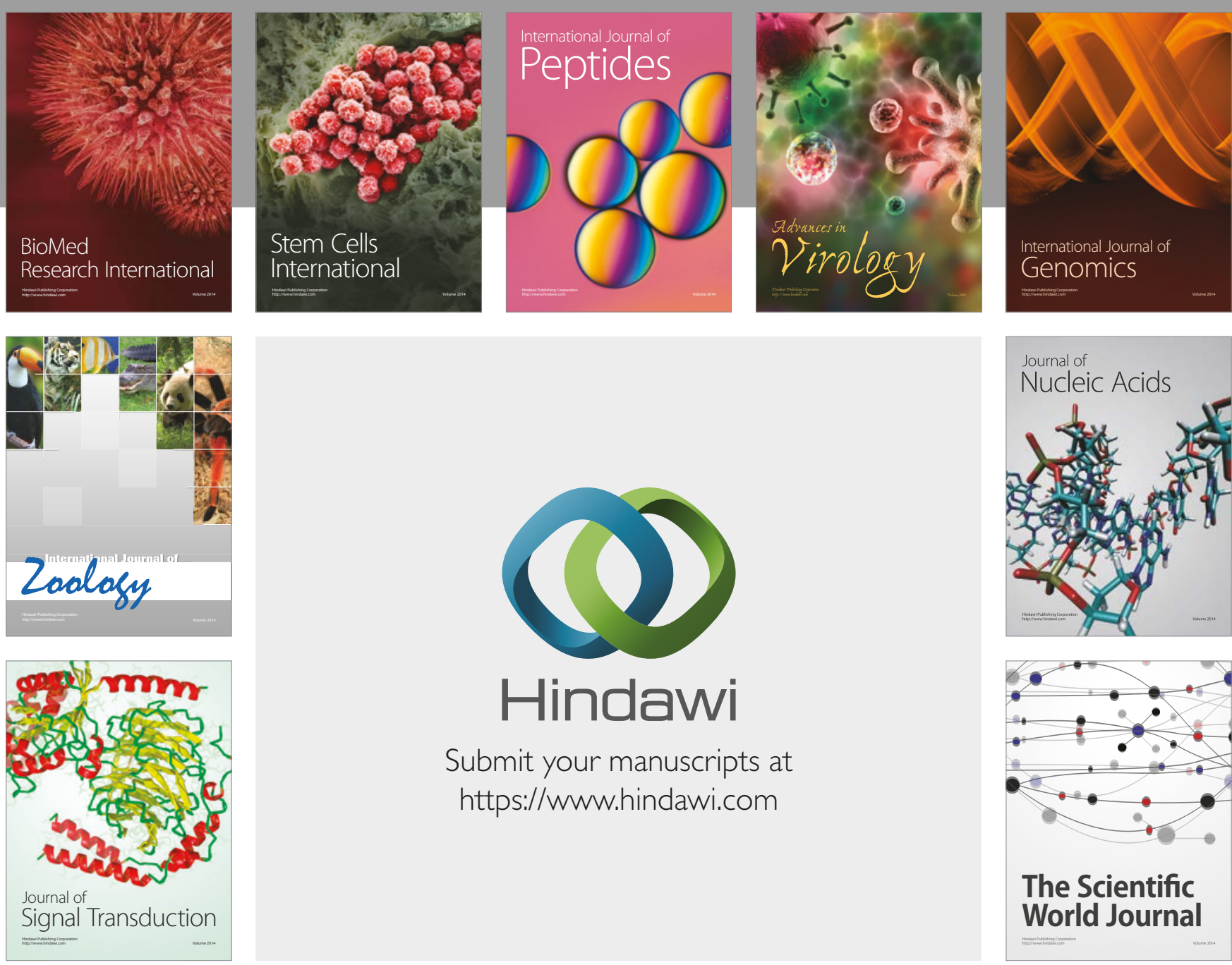

Submit your manuscripts at

https://www.hindawi.com
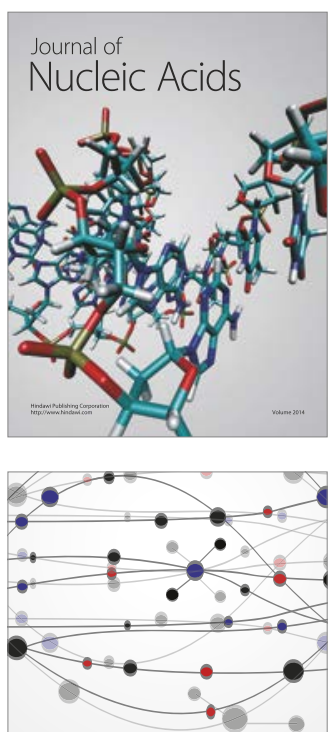

The Scientific World Journal

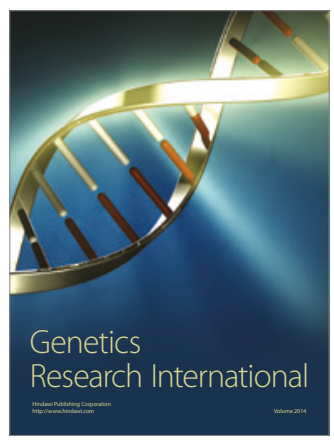

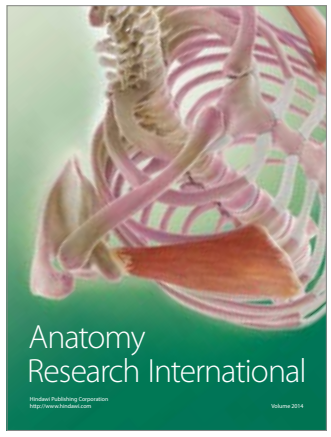

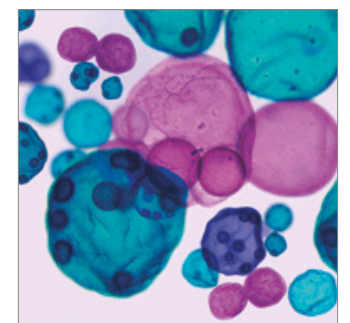

International Journal of Microbiology
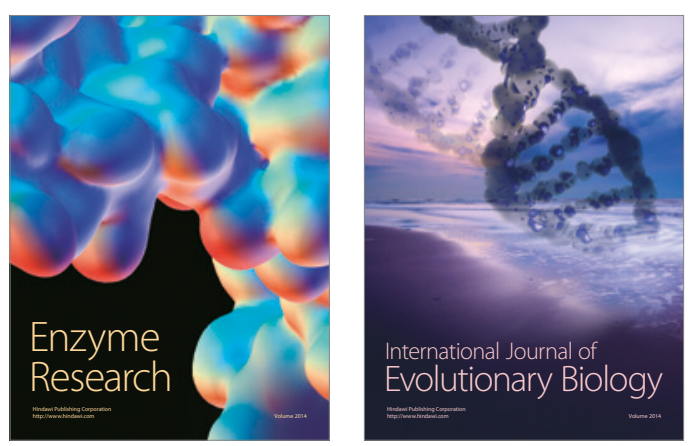
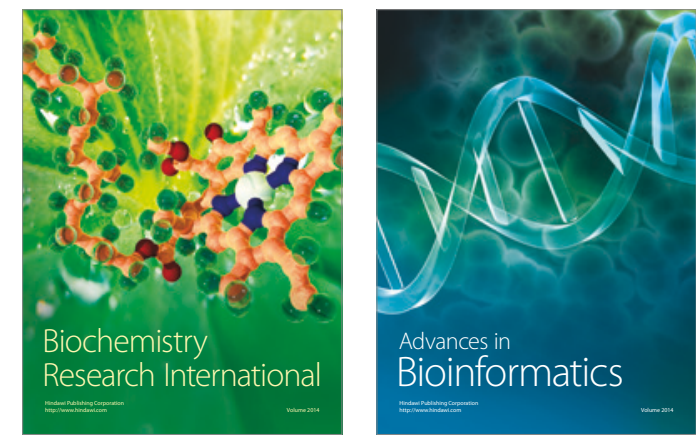

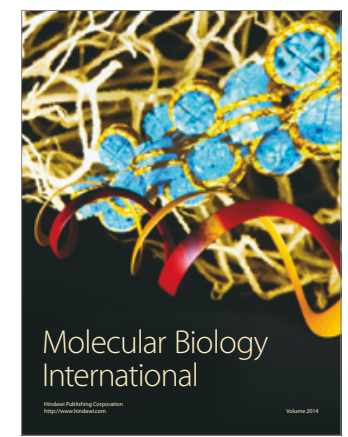

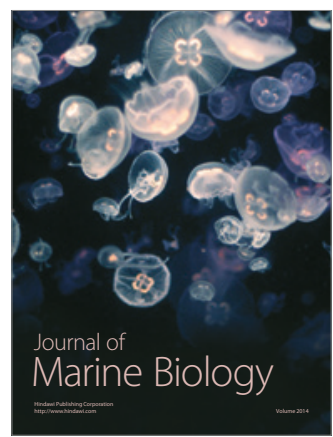

